\title{
Circulating Tumor Cell Detection In Epithelial Ovarian Cancer Using Dual-Component Antibodies Targeting EpCAM And FR $\alpha$
}

This article was published in the following Dove Press journal: Cancer Management and Research

Na Li D'
Hao Zuo'
Luojun Chen (D'
Huali Liu'
Jin Zhou'
Yi Yao ID'
Bin Xu ID'
Hongyun Gong (D)
Yiming Weng'
Qinyong Hu'
Qibin Song'
Min Peng'
Yanxiang Cheng ${ }^{2}$
'Department of Oncology, Renmin
Hospital of Wuhan University, Wuhan,
Hubei, People's Republic of China;
'Department of Obstetrics and
Gynecology, Renmin Hospital of Wuhan
University, Wuhan, Hubei, People's
Republic of China

Correspondence: Min Peng Department of Oncology, Renmin Hospital of Wuhan University, Wuhan, Hubei 430060, People's Republic of China Tel +86 I33 17/3 3/40

Email mpeng320@whu.edu.cn

Yanxiang Cheng

Department of Obstetrics and

Gynecology, Renmin Hospital of Wuhan

University, Wuhan, Hubei 430060,

People's Republic of China

Tel +86278804 I9II

Email doctornancy@qq.com
Purpose: Circulating tumor cell (CTC) detection methods based on epithelial cell adhesion molecule (EpCAM) have low detection rates in epithelial ovarian cancer (EOC). Meanwhile, folate receptor alpha $(\mathrm{FR} \alpha)$ has high expression in EOC cells. We explored the feasibility of combining FR $\alpha$ and EpCAM as CTC capture targets in EOC.

Patients and methods: EpCAM and FR $\alpha$ antibodies were linked to magnetic nanospheres (MNs) using the principle of carbodiimide chemistry. Blood samples from healthy donor spiked with A2780 ovarian cancer cells were used for detecting the capture rate. Ninety-five blood samples from 30 patients with EOC were used for comparing the positive rate of detection when using anti-EpCAM-MNs alone with that when using combination of antiEpCAM-MNs and anti-FR $\alpha$-MNs. Samples from 28 patients initially diagnosed with EOC and 20 patients with ovarian benign disease were used for evaluating the sensitivity and specificity of combination of anti-EpCAM-MNs and anti-FR $\alpha$-MNs.

Results: Regression analysis between the number of recovered and that of spiked A2780 cells revealed $\mathrm{y}_{\text {EpCAM }}=0.535 \mathrm{x}\left(\mathrm{R}^{2}=0.99\right), \mathrm{y}_{\mathrm{FR} \alpha}=0.901 \mathrm{x}\left(\mathrm{R}^{2}=0.99\right)$, and $\mathrm{y}_{\mathrm{EPCAM}+\mathrm{FR} \alpha}=$ $0.928 x\left(R^{2}=0.99\right)$. In mixtures of $A 2780$ and MCF7 cells, the capture rate was $92 \%$ using the combination of anti-EpCAM-MNs and anti-FR $\alpha-M N s$, exceeding the rate when using anti-EpCAM-MNs or anti-FR $\alpha$-MNs alone by approximately $20 \%(\mathrm{P}<0.01)$. The combination of anti-EpCAM-MNs and anti-FR $\alpha$-MNs showed a significantly increased positive rate of CTC detection in EOC patients compared with anti-EpCAM-MNs alone $\left(\chi^{2}=14.45, \mathrm{P}<\right.$ 0.001 ). Sensitivity values were 0.536 and 0.75 and specificity values were 0.9 and 0.85 when using anti-EpCAM-MNs alone and when using the combination of anti-EpCAM-MNs and anti-FR $\alpha-\mathrm{MNs}$, respectively.

Conclusion: The combination of FR $\alpha$ and EpCAM is feasible as a CTC capture target of CTC detection in patients with EOC.

Keywords: circulating tumor cells, ovarian cancer, epithelial cell adhesion molecule, folate receptor alpha

\section{Introduction}

Epithelial ovarian cancer (EOC) is one of the deadliest gynecological malignancies, accounting for $2.5 \%$ of all malignancies among females, but $5 \%$ of cancer-related deaths in this population. ${ }^{1}$ Owing to the lack of sensitive signs and symptoms and effective screening methods, most patients are diagnosed at stage III (51\%) or IV $(29 \%){ }^{1}$ Although survival has improved with the use of cytoreductive surgery along with platinum- and taxane-based chemotherapy, nearly $80 \%$ of patients with ovarian cancer eventually relapse within 5 years. ${ }^{1}$ Therefore, EOC detection 
in the early stage and monitoring of tumor progression are extremely important for improving patient outcomes.

Circulating tumor cells (CTCs), which are shed from the tumor into the bloodstream, are emerging as novel diagnostic or prognostic biomarkers for solid tumors, including EOC. ${ }^{2}$ However, CTCs are extremely rare in an extremely complex matrix of blood, requiring a detection method with high sensitivity and specificity. Some detection methods, such as immunomagnetic separation, ${ }^{3}$ microfluidic separation, ${ }^{4}$ filter-based methods, ${ }^{5}$ and ligandtargeted $\mathrm{PCR}^{6}$ have been developed in recent years. Each detection method has limitations because of heterogeneity of CTCs. Among these platforms, the CellSearch system (Veridex, Raritan, NJ, USA) is the first US Food and Drug Administration (US FDA)-approved test for capturing and enumerating CTCs of breast, prostate, or colorectal cancer using anti-epithelial cell adhesion molecule (EpCAM) coated magnetic beads. ${ }^{3}$ However, because of low EpCAM expression or epithelial-to-mesenchymal transition (EMT) during metastasis in EOC, EpCAM-based enrichment has low sensitivity for CTC detection in patients with EOC. ${ }^{2}$ Thus, the selection of highly expressed EOC-specific antigens on the cell surface of CTCs is important for improving the CTC detection rate.

Folate receptor alpha $(\mathrm{FR} \alpha)$, which is a glycosylated phosphatidylinositol-anchored glycoprotein that binds to folic acid, has high expression in EOC. Studies have reported that $>95 \%$ of patients with EOC overexpress FR $\alpha$ on the cell membrane, whereas the receptor is expressed at extremely low levels in most healthy tissues. ${ }^{7,8}$ Thus, FR $\alpha$ is an ideal immune capture target for CTC detection in patients with EOC.

Combining different immune capture targets can improve the CTC detection rate. ${ }^{9-11}$ Thus, we combined $\mathrm{FR} \alpha$ and EpCAM as capture targets for CTC detection in EOC. In this study, we demonstrated higher efficacy and sensitivity of the combination of EpCAM and FR $\alpha$ as capture targets in EOC cell lines and patients with EOC, suggesting their translational potential for future development in CTC detection methods.

\section{Materials And Methods}

\section{Cell Culture}

A2780 cell (human EOC), MCF7 cell (human breast cancer), A549 cell (human non-small cell lung cancer), and Jurkat T-cell (human T-cell leukemia) lines were purchased from the China Center for Type Culture Collection. MCF7 cells were cultured in DMEM (Life Technologies, Carlsbad, USA) with 10\% FBS (ScienCell, Carlsbad, CA, USA), and the other cells were cultured in RPMI 1640 (Life Technologies) with $10 \% \mathrm{FBS}$. All cells were cultured at $37^{\circ} \mathrm{C}$ in a humidified atmosphere containing $5 \% \mathrm{CO}_{2}$.

\section{Flow Cytometry And Immunofluorescence Staining}

Flow cytometry was used for analyzing EpCAM and FR $\alpha$ expression on the membrane of A2780 cells. Cells were detached using $0.25 \%$ trypsin-EDTA (Life Technologies), after which $1 \times 10^{6}$ cells were resuspended in a $10-\mathrm{mL}$ EP tube and fixed with $4 \%$ paraformaldehyde (Servicebio, Wuhan, China). After washing with PBS, cells blocked with 2\% BSA (Servicebio) and incubated with anti-EpCAM (SAB4700423, Sigma-Aldrich, St. Louis, MO, USA) or antiFR $\alpha$ (MAB5646, R\&D Systems, Minneapolis, MN, USA) monoclonal antibody for $30 \mathrm{mins}$ at $37^{\circ} \mathrm{C}$. After washing with PBST, the cells were incubated with Alexa Fluor ${ }^{\circledR} 488$ conjugated donkey anti-mouse $\operatorname{IgG}(\mathrm{H}+\mathrm{L})$ secondary antibody (A21202, Life Technologies). Isotype controls were incubated with irrelevant mouse IgG primary and secondary antibodies, and the negative control was not treated after washing with PBS. The fluorescence signal was detected using three-laser FACSCalibur (BD Biosciences, San Jose, CA, USA) and analyzed using FlowJo software, version 10.0 (TreeStar Inc.).

For immunofluorescence, A2780 cells grown on coverslips were fixed using $4 \%$ paraformaldehyde for 10 mins, blocked using 2\% BSA for $30 \mathrm{mins}$, and then processed using anti-EpCAM and anti-FR $\alpha$ monoclonal antibodies overnight at $4^{\circ} \mathrm{C}$. After thorough washing with PBST $(3 \times 5$ mins $)$, the cells were incubated with Alexa Fluor 488-conjugated donkey anti-mouse $\operatorname{IgG}(\mathrm{H}+\mathrm{L})$ secondary antibody. Nuclei were visualized using DAPI (Servicebio). After thorough washing with PBST, cells were coverslipped using antifade mounting medium (Servicebio). As a negative control, the primary antibody was omitted, and all other steps were performed as described previously. Fluorescence images were photographed using a fluorescence microscope (BX63, Olympus, Japan).

Construction Of Anti-EpCAM And AntiFR $\alpha$ Antibody-Modified Magnetic

\section{Nanospheres}

The principle of carbodiimide chemistry was used for cross-linking the antibodies with magnetic nanospheres 
(MNs). Specifically, $10 \mathrm{mg}$ of Sera-Mag ${ }^{\circledR}$ speedBeads $^{\mathrm{TM}}$ MNs (1 mg/mL, Sigma-Aldrich) were washed twice with PBS (10 mM, pH 6.8) and dispersed into $1000 \mu \mathrm{L}$ of PBS (10 mM, pH 6.8), after which the samples were incubated with $10 \mathrm{mg} / 200 \mu \mathrm{L}$ of EDC (Sigma-Aldrich) and $5 \mathrm{mg} / 200$ $\mu \mathrm{L}$ of NHS (Sigma-Aldrich) for 30 mins to activate the carboxyl groups on the surface of the functional nanospheres. Then, the nanospheres were washed twice with PBS (10 mM, pH 7.2), dispersed in $1000 \mu \mathrm{L}$ of PBS (10 $\mathrm{mM}, \mathrm{pH}$ 7.2), and reacted with EpCAM or FR $\alpha$ antibody for $4 \mathrm{hrs}$ with continuous shaking at $37 \mathrm{C}$. After the reaction was completed, the antibody-modified nanospheres were washed with PBS (10 mM, pH 7.2) and finally stored in PBS (containing $0.05 \% \mathrm{NaN}_{3}$ and $1 \%$ BSA) at $4^{\circ} \mathrm{C}$.

Immunofluorescence was used to verify binding between the antibody and magnetic beads. Specifically, $2 \mu \mathrm{L}$ of antiEpCAM-MNs or anti-FR $\alpha-M N s$ was washed twice with PBS, incubated with Alexa Fluor 488-conjugated donkey anti-mouse IgG $(\mathrm{H}+\mathrm{L})$ secondary antibody for $30 \mathrm{mins}$ at $37^{\circ} \mathrm{C}$, washed with PBST, and coated onto a slide. Fluorescence images were photographed using a fluorescence microscope (BX63, Olympus).

\section{Capture Of Spiked Tumor Cells In PBS, Lysed Blood, And Whole Blood}

Hoechst 33342-stained A2780 (EpCAM ${ }^{\text {low }} / \mathrm{FR}^{\text {high }}{ }^{\text {) cells }}$ were added to PBS, lysed blood, and whole blood at a concentration of 200 cells $/ \mathrm{mL}$ to prepare closely mimicking clinical samples. As controls, immunomagnetic nanospheres (IMNs) were used to capture MCF7 cells (EpCAM ${ }^{\text {high} / ~}$ $\left.\mathrm{FR}^{\text {low }}\right)$, A549 cells $\left(\mathrm{EpCAM}^{\mathrm{low}} / \mathrm{FR} \alpha^{-}\right)$, and Jurkat T-cells $\left(\mathrm{EpCAM}^{-} / \mathrm{FR} \alpha^{-}\right)$and unmodified MNs were used to capture cells to investigate the specificity of the modified MNs. To prove that high enrichment efficiency was achieved via the capture of $\mathrm{EpCAM}^{+}$and $\mathrm{FR}^{+}$cells, we used a mixture of A2780 and MCF7 cells. Approximately $0.15 \mathrm{mg} / \mathrm{mL}$ IMNs were added to the aforementioned samples, and they were incubated at $37^{\circ} \mathrm{C}$ for $15 \mathrm{mins}$. Then, they were isolated and washed with a magnetic scaffold. After thorough washing with PBS, the captured cells were counted under a fluorescence microscope. Captured and uncaptured cells were all counted to calculate the capture efficiency. Each experiment was repeated three times.

We tested the ability of IMNs to capture rare A2780 cells in synthetic CTC samples, which were prepared by spiking stained A2780 cells into whole blood at concentrations of 5,
50, 100, 200, and 300 cells $/ \mathrm{mL}$. Approximately $0.15 \mathrm{mg} / \mathrm{mL}$ IMNs were added to the samples, and they were incubated at $37^{\circ} \mathrm{C}$ for 15 mins, isolated, and washed with a magnetic scaffold. After thorough washing with PBS, the captured cells were counted under a fluorescence microscope.

\section{ICC Identification And Viability Of The Captured Cells In Mimicked Clinical Samples} A2780 cells were spiked into whole blood at a concentration of approximately 200 cells $/ \mathrm{mL}$ to prepare mimicking clinical samples. The mimicking clinical samples were incubated with IMNs for 15 mins. After magnetic separation, the captured cells were fixed with $4 \%$ paraformaldehyde (10 mins); permeabilized with $0.1 \%$ Triton X-100 (10 mins); blocked with 2\% BSA (30 mins); and incubated with Alexa Fluor 568-labeled anti-cytokeratin 19 (CK19) monoclonal antibody, Alexa Fluor 488-labeled anti-CD45 monoclonal antibody, and DAPI for 30 mins. After thorough washing with PBS, the cells were coverslipped using antifade mounting medium. Fluorescence images were obtained using a fluorescence microscope BX63 (Figure 1B). Cells with CK19-positive and DAPI-positive but CD45-negative phenotypes were enumerated as CTCs.

A Live/Dead viability kit was used for analyzing the viability of the captured cells. Cells were stained with calcein-AM and propidium iodide (PI). The final concentration of calcein-AM and PI was 2 and $4 \mu \mathrm{mol} / \mathrm{L}$, respectively. Then, the cells were observed under a fluorescence microscope, and five fields were randomly selected to calculate the viability rate.

\section{CTC Detection In Patients With EOC}

All experimental protocols were approved by the ethics committee of Renmin Hospital of Wuhan University. All experiments were performed in accordance with relevant guidelines and regulations.

Whole blood samples $(8 \mathrm{~mL})$ were collected in EDTA tubes (BD Biosciences) and used within $24 \mathrm{hrs}$. All blood samples were divided into two tubes $(4 \mathrm{~mL})$ and captured using anti-EpCAM-MNs or a combination of antiEpCAM-MNs and anti-FR $\alpha-M N s$. CTCs were denoted by CK19 and DAPI positivity and CD45 negativity. In total, blood samples were collected from 30 patients with EOC undergoing surgery or chemotherapy and 20 patients with benign ovarian diseases after obtaining informed consent. Among the 30 ovarian cancer patients, blood samples were taken once or up to eight times before 
A

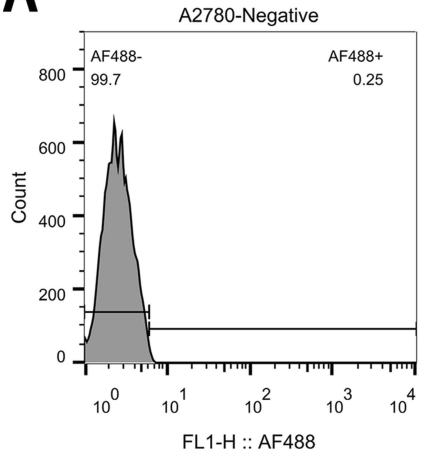

B

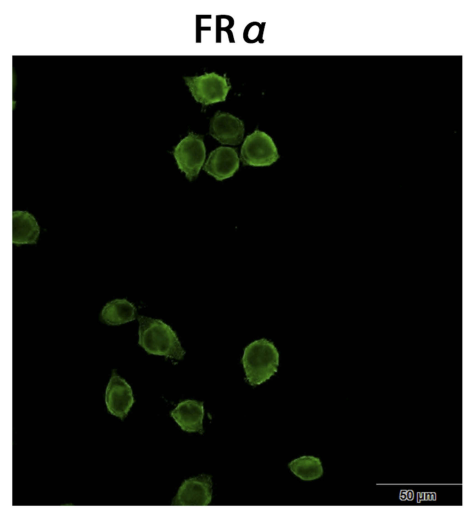

EpCAM

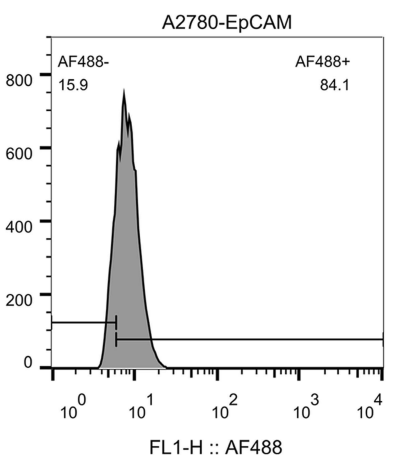

Merge

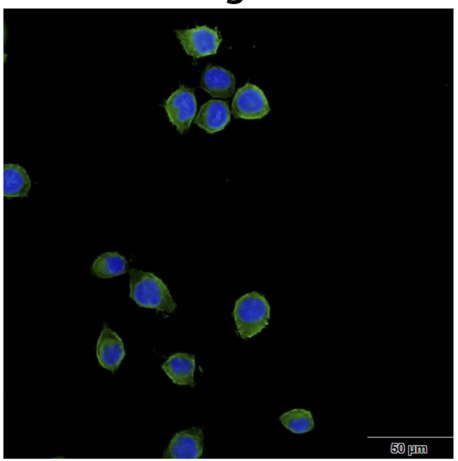

Merge

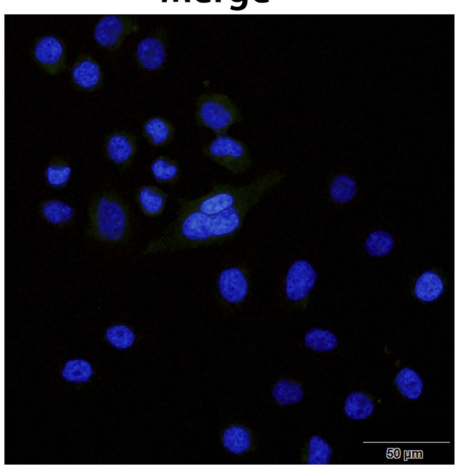

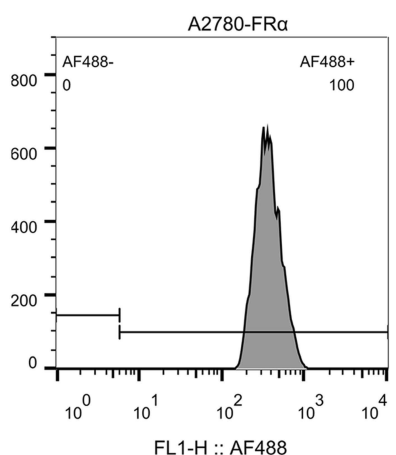

c

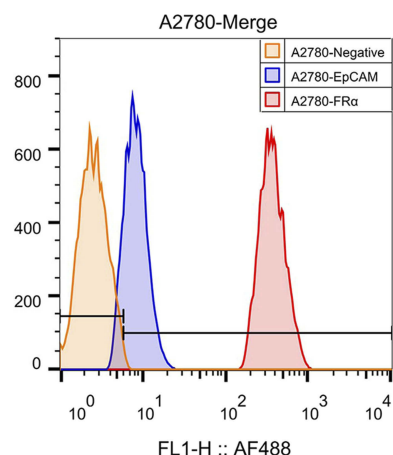

FL1-H :: AF488
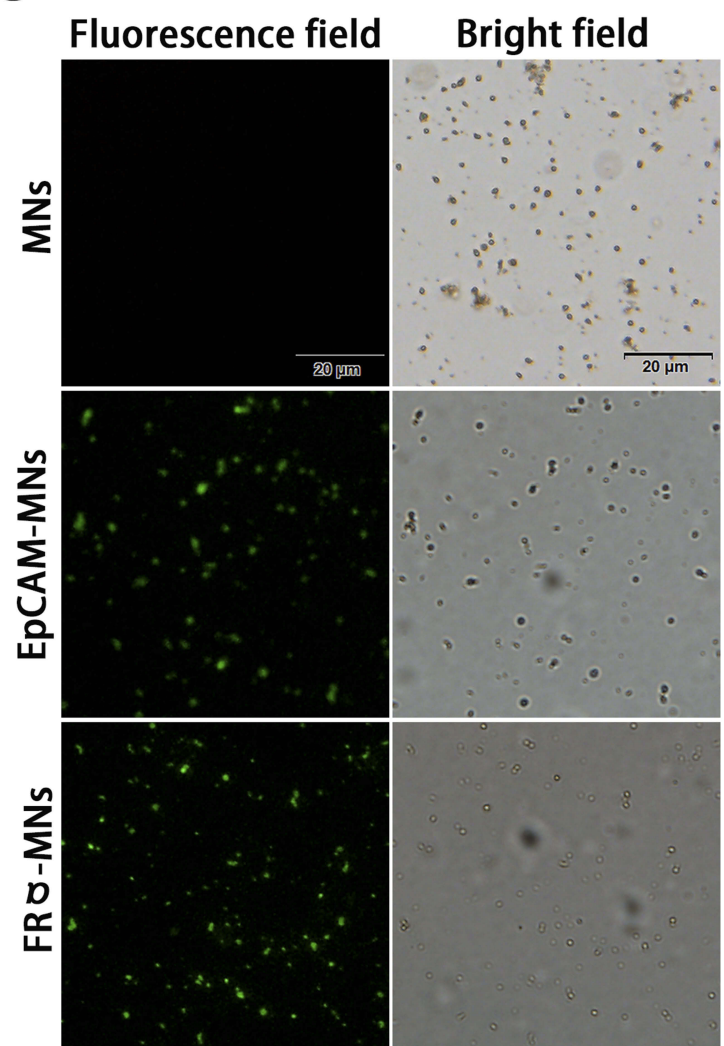

Figure I Expression of EpCAM and FR $\alpha$ in A2780 cells and identification of synthesized MNs. (A) Flow cytometry detection of epithelial cell adhesion molecule (EpCAM) and folate receptor alpha (FR $\alpha$ ) expression in A2780 cells. A2780 cells were stained with anti-EpCAM (blue) and anti-FR $\alpha$ antibodies (red) and the negative control was autofluorescent (orange). (B) Immunofluorescence detection of EpCAM and FR $\alpha$ expression in A2780 cells. EpCAM and FR $\alpha$ stained with Alexa Fluor ${ }^{\circledR} 488$ are green at an excitation wavelength of $488 \mathrm{~nm}$, and nuclei stained with DAPI are blue at an excitation wavelength of $405 \mathrm{~nm}$. (C) Identification of synthesized magnetic nanospheres. The Alexa Fluor 488-conjugated donkey anti-mouse $\lg G(\mathrm{H}+\mathrm{L})$ secondary antibody specifically bound to antibody-modified magnetic beads (green).

surgery or each cycle of chemotherapy, with an average of 3.2 times and a median of 2 times.

\section{Statistical Analysis}

Statistical analysis was performed using SPSS software, version 24.0 (SPSS Inc., Armonk, USA). The number of CTCs in different CTC detection method groups was compared using a two-tailed Student's $t$-test. A paired chi-square test was used for evaluating the difference between the two detection rates. A two-sided $P<0.05$ was considered to be statistically significant.

\section{Results}

Expression Of EpCAM And FR $\alpha$ In A2780 Cells And Identification Of Synthesized

\section{MNs}

EpCAM and FR $\alpha$ expression in A2780 cells were analyzed using flow cytometry. The results illustrated that 
FR $\alpha$ expression was high in A2780 cells, and the binding rate of the antibody to cells was $99.7 \%$; EpCAM expression was low, and the binding rate of the antibody to cells was $84.1 \%$ (Figure 1A). Immunofluorescence also confirmed this result. Specifically, FR $\alpha$ shows high expression on the A2780 cell membrane, whereas EpCAM shows low expression (Figure 1B). The Alexa Fluor 488-conjugated donkey anti-mouse $\mathrm{IgG}(\mathrm{H}+\mathrm{L})$ secondary antibody specifically bound to antibody-modified MNs, demonstrating successful binding of the antibody to MNs (Figure 1C).

\section{Ability Of IMNs To Capture Target Cells}

First, we tested the ability of IMNs to capture A2780 cells in synthetic CTC samples, the capture rate of anti-FR $\alpha$ MNs to capture A2780 cells was 90.0\%, 86.7\%, 85.9\%, respectively, the capture rate of anti-EpCAM-MNs to capture A2780 cells was 43.4\%, 41.9\%, 39.1\%, respectively, the capture rate of combination of anti-EpCAM-MNs and anti-FR $\alpha$-MNs to capture A2780 cells was $92.5 \%, 89.5 \%$, $88.6 \%$, respectively (Figure $2 \mathrm{~A}$ ). The results indicated that anti-FR $\alpha$-MNs can significantly improve CTC enrichment efficiency compared with anti-EpCAM-MNs $(\mathrm{P}<0.001)$. The efficiency of the test was virtually unaffected by the liquid environment.

Then, we tested the specificity of the detection methods. We used IMNs to capture A2780 cells (EpCAM ${ }^{\text {low/ }}$ FR $\left.\alpha^{\text {high }}\right)$, MCF7 cells $\left(\right.$ EpCAM $\left.{ }^{\text {high }} / \mathrm{FR} \alpha^{\text {low }}\right)$, A549 cells $\left(\mathrm{EpCAM}^{\text {low }} / \mathrm{FR} \alpha^{-}\right)$, and Jurkat T-cells $\left(\mathrm{EpCAM}^{-} / \mathrm{FR} \alpha^{-}\right)$ (Figure 2B). Anti-EpCAM-MNs could capture EpCAMexpressing cells and anti-FR $\alpha-\mathrm{MNs}$ could capture FR $\alpha$ expressing cells, the capture rate was more than $88 \%$. Neither type of MNs could capture Jurkat T-cells, the capture rate was less than $6.6 \%$. Thus, IMNs modified with different antibodies can only capture the target cells of the corresponding antigen. Unmodified MNs could hardly capture target cell including A2780 cells, MCF7 cells, A549 cells and Jurkat T cells, and the capture rate was less than $6.9 \%$. These results demonstrated that IMNs have good specificity.

To demonstrate that high enrichment efficiency was achieved by the capture of $\mathrm{EpCAM}^{+}$and $\mathrm{FR}^{+}$cells, we used a mixture of $\mathrm{A} 2780$ cells $\left(\mathrm{EpCAM}^{\text {low }} / \mathrm{FR}^{\text {high }}\right)$ and MCF7 cells $\left(\mathrm{EpCAM}^{\mathrm{high}} / \mathrm{FR} \alpha^{\text {low }}\right)$ cells. In the mixed cell samples, the capture rate was more than $92.5 \%$ using a combination of anti-EpCAM-MNs and anti-FR $\alpha-M N s$, which exceeded the rate when using anti-EpCAM-MNs or anti-FR $\alpha$-MNs alone by approximately $20 \%(\mathrm{P}<0.01)$ (Figure 2C). Therefore, we demonstrated that the enrichment efficiency was improved by the capture of two antigens (EpCAM and $\mathrm{FR} \alpha$ ) expressed on the cell membrane.

We tested the ability of IMNs to capture rare A2780 cells in synthetic CTC samples. When the concentration of the mixed cells ranges from 5 to 300 cells, the relationship between the numbers of recovered and spiked tumor cells was linear, and the regression analysis produced $\mathrm{У}_{\mathrm{EpCAM}}$ $=0.535 \times\left(R^{2}=0.99,95 \% \mathrm{CI}=0.523-0.547\right)($ Figure $2 \mathrm{D})$, $\mathrm{y}_{\mathrm{FR} \alpha}=0.901 \mathrm{x} \quad\left(\mathrm{R}^{2}=0.99,95 \%\right.$ CI $\left.=0.889-0.913\right)$ (Figure 2E), and $\mathrm{y}_{\mathrm{EpCAM}+\mathrm{FR} \alpha}=0.928 \mathrm{x}\left(\mathrm{R}^{2}=0.99,95 \%\right.$ $\mathrm{CI}=0.914-0.942)$ (Figure $2 \mathrm{~F})$. Therefore, it can be concluded that IMNs can efficiently and specifically capture rare target tumor cells from whole blood.

\section{ICC Identification And Viability Of The Captured Cells}

A three-color ICC method including AF488-labeled antiCD45, AF594-labeled anti-CK19, and DAPI was used for identifying the captured cells. CK19 is a marker for epithelial cells, and CD45 is a marker for white blood cells. CK19positive (red), DAPI-positive (blue), and CD45-negative cells (green) were enumerated as CTCs (Figure 2G).

Calcein-AM and PI were used for staining cells to analyze the capture ability. Calcein-AM can penetrate the live cell membrane and react with intracellular esterase to form calcein with green fluorescence, whereas PI is a membraneimpermeable nuclear stain that can stain only dead cells with red fluorescence. As shown in Figure $2 \mathrm{H}$, majority of the isolated cells exhibited green fluorescence, and the viability rate was $92 \% \pm 1.8$, indicating that most of the tumor cells remained viable after isolation. The bright field photographs illustrate that the integrity of the cells was good and that the IMNs bound to the surface of the cells (Figure 2I).

\section{CTC Detection In Peripheral Blood Samples From Patients With EOC}

In total, Ninety-five blood samples were collected from 30 patients with EOC undergoing surgery or chemotherapy and 20 patients with benign ovarian diseases after obtaining written informed consent. Patient characteristics are shown in Table 1 . Among the 30 ovarian cancer patients, blood samples were taken once or up to eight times before surgery or each cycle of chemotherapy, with an average of 3.2 times and a median of 2 times, each samples were tested using antiEpCAM-MNs alone or using a combination of antiEpCAM-MNs and anti-FR $\alpha$-MNs. CTC levels in the two groups, as measured using each method, are plotted in 
A

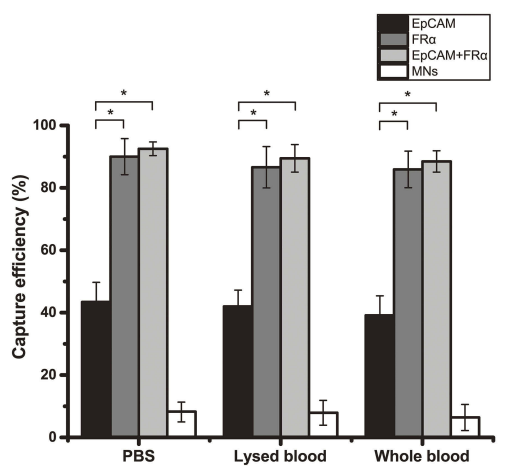

D

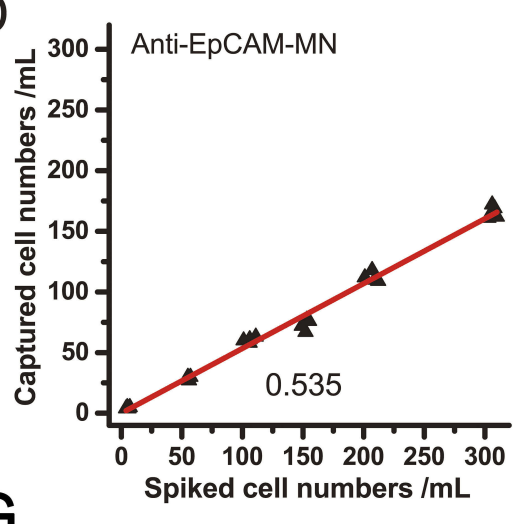

G

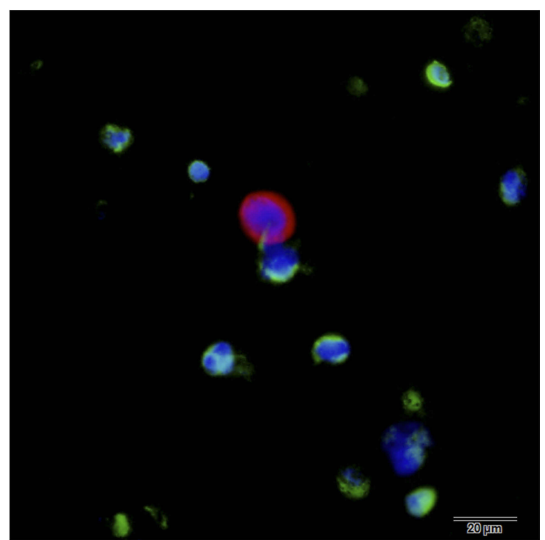

B

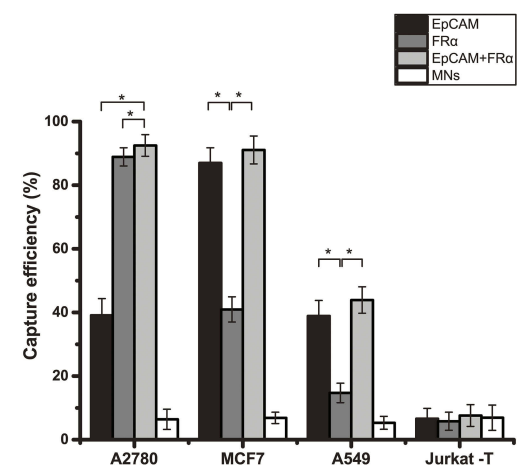

E

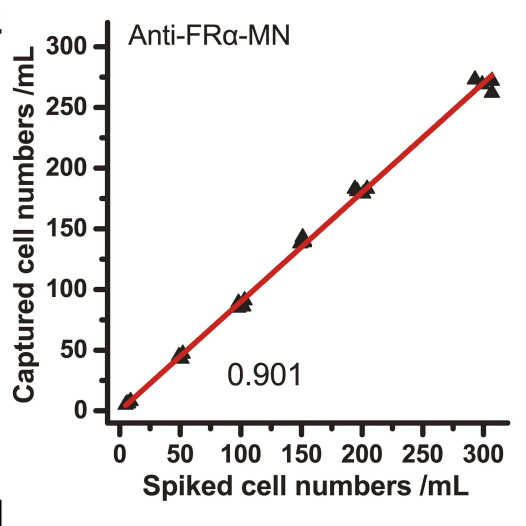

H

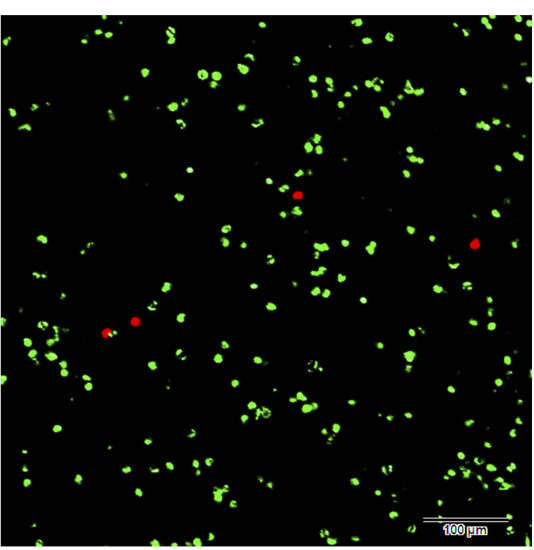

C

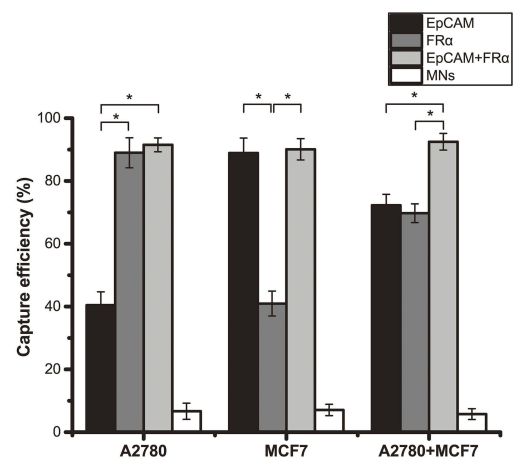

F
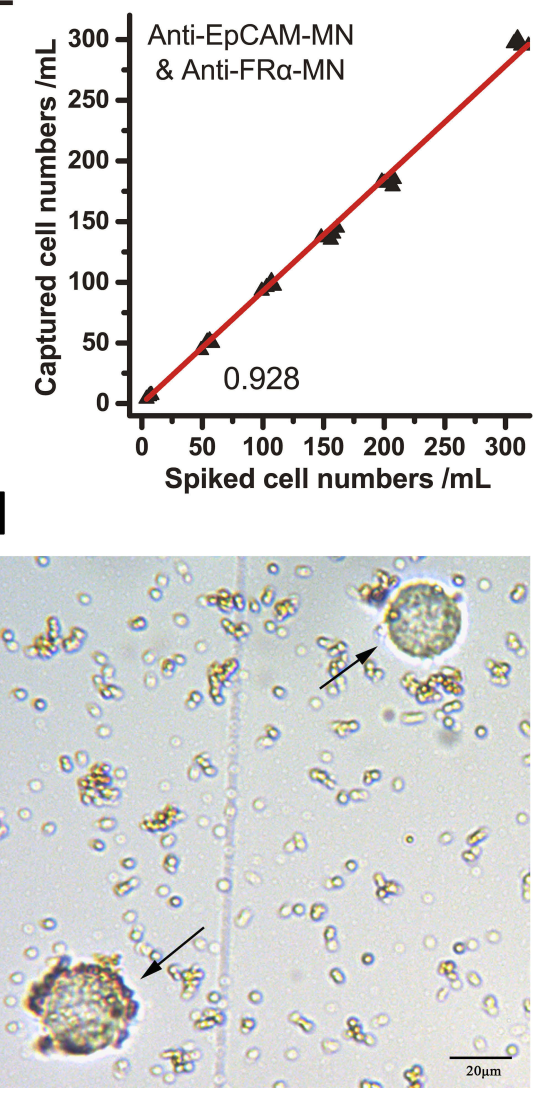

Figure 2 Efficiencies of anti-EpCAM-MNs and anti-FR $\alpha-M N s$ used alone or in combination to capture target cells. (A) Capture efficiencies of using anti-EpCAM-MNs alone, anti-FR $\alpha$-MNs alone, or a combination of both to capture A2780 cells in PBS, lysed blood, and whole blood, and unmodified MNs served as a negative control. (B) Capture efficiencies of using anti-EpCAM-MNs alone, anti-FRa-MNs alone, or a combination of both for capturing A2780 cells (EpCAM ${ }^{\text {low }} / \mathrm{FR}^{\text {high }}$ ), MCF7 cells $\left(\mathrm{EpCAM}^{\text {high }} / \mathrm{FR} \alpha^{\text {low }}\right), \mathrm{A} 549$ cells $\left(\mathrm{EpCAM}{ }^{\text {low }} / \mathrm{FR} \alpha^{-}\right)$, and Jurkat T-cells (EpCAM $\left.{ }^{-} / \mathrm{FR} \alpha^{-}\right)$. (C) Capture efficiencies of anti-EpCAM-MNs (light gray), anti-FR $\alpha-M N s$ (dark gray), combination of anti-EpCAM-MNs and anti-FR $\alpha-M N s$ (white), and unmodified MNs (black) to capture A2780, MCF7, or a mixture of A2780 and MCF7 cells. (D, E, F) The regression analysis plots of recovered vs spiked A2780 cells detected using anti-EpCAM-MNs (D), anti-FRa-MNs (E), and a combination of anti-EpCAM-MNs and anti-FRa-MNs (F). (G) Three-color ICC identified the capture cells. CTCs were CKI9-positive (red), DAPI-positive (blue), and CD45-negative (green). (H) Fluorescence microscopic image of the captured cells stained with calcein-AM (green) and propidium iodide (red). (I) Bright field photograph of the captured cells shows that the cells remain intact (arrows). Each experiment was repeated three times. $* \mathrm{P}<0.05$.

Figure 3A; the results from the same patient with EOC are shown in Figure 3B. The CTC level in patients with EOC was $0-18$ when using anti-EpCAM-MNs and $0-32$ when using the combination of anti-EpCAM-MNs and anti-FR $\alpha$-MNs. The
CTC level was $0-2$ for patients with benign diseases. The area under the receiver operating characteristic curve (AUC-ROC) analysis indicated that the combination of anti-EpCAM-MNs and anti-FR $\alpha-M N s$ had a higher AUC (0.8011, 95\% CI: 
Table I Patient Characteristics

\begin{tabular}{|l|l|l|}
\hline Characteristic & N & Percent \\
\hline Total & 30 & $100.0 \%$ \\
Initial & 28 & $93.3 \%$ \\
Relapse & 2 & $6.7 \%$ \\
\hline Histological type & & \\
Serous carcinoma & 25 & $83.3 \%$ \\
Mucinous carcinoma & 2 & $6.7 \%$ \\
Clear cell carcinoma & 3 & $10.0 \%$ \\
\hline AJCC stage & & \\
I-II & 9 & $30.0 \%$ \\
III-IV & 21 & $70.0 \%$ \\
\hline Grade & & \\
Low-grade & 7 & $23.3 \%$ \\
Borderline & 2 & $6.7 \%$ \\
High-grade & 18 & $60.0 \%$ \\
Unknown & 3 & $10.0 \%$ \\
\hline
\end{tabular}

0.7212-0.8809, $\mathrm{P}<0.001)$ than anti-EpCAM-MNs alone (0.7208, 95\% CI: $0.6209-0.8206, \mathrm{P}=0.002)$. When the cutoff value was set to 2 (CTC number $\geq 2$ is CTC positive and CTC number $<2$ is CTC negative), the highest Youden's index was achieved. At this cut-off, the positive rate was $48.42 \%$ using anti-EpCAM-MNs alone and 67.36\% using the combination of anti-EpCAM-MNs and anti-FR $\alpha-M N s$. A paired chi-square test revealed a significant difference between the two methods $\left(\chi^{2}=14.45, \mathrm{P}<0.001\right)$ (Table 2$)$. An image gallery of representative CTCs from patients with EOC is shown in Figure 3C.

\section{Sensitivity And Specificity Of CTC} Detection In Patients With Suspected EOC Of the 30 patients with EOC, 28 were initially diagnosed with EOC. The number of CTCs tested separately and jointly before surgery in 28 patients with EOC and 20
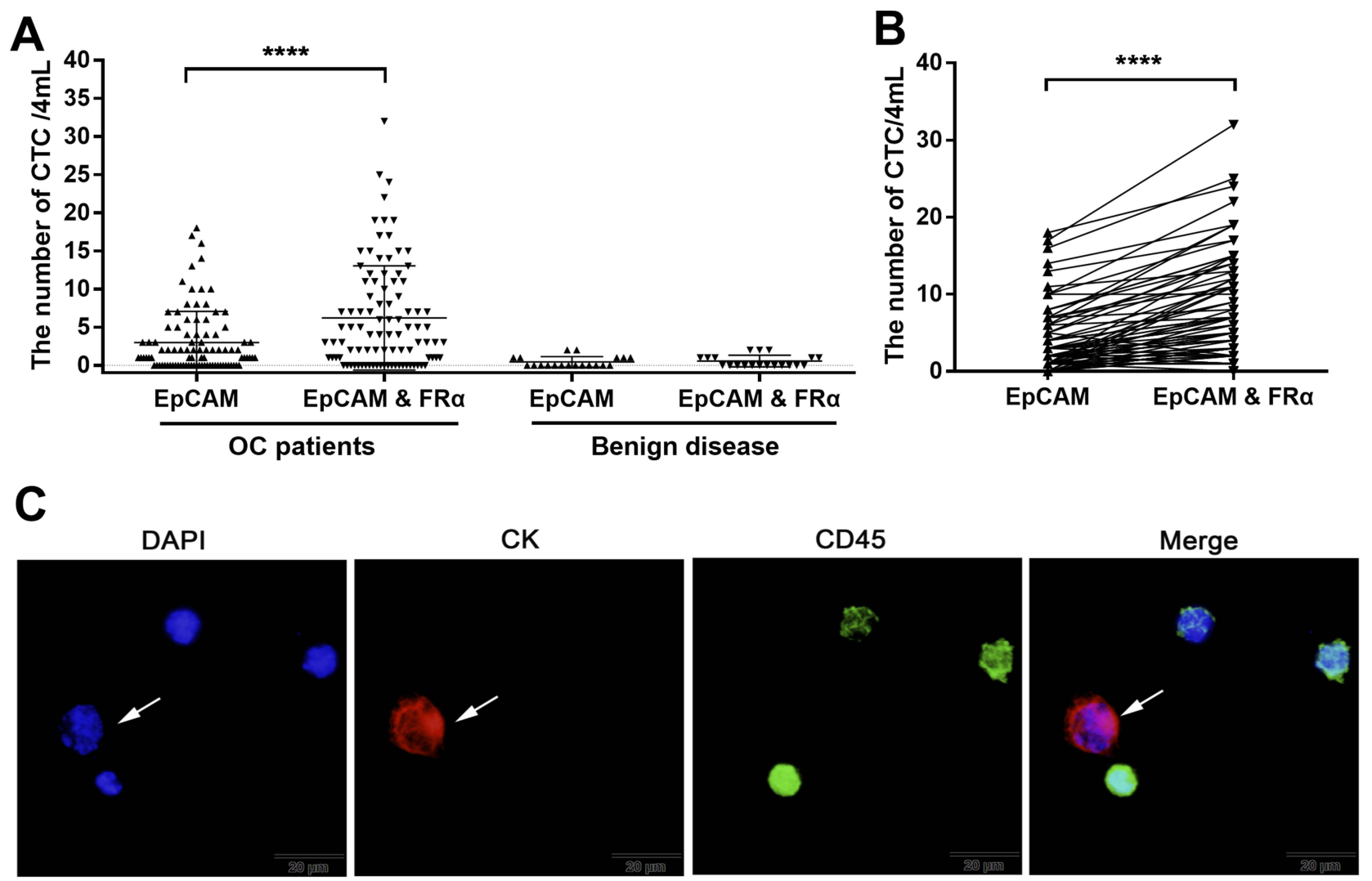

Figure 3 CTC detection in peripheral blood samples from patients with EOC. (A) CTC levels in 95 blood samples from 30 patients with EOC and 20 patients with benign disease as identified using anti-EpCAM-MNs alone or a combination of anti-EpCAM-MNs and anti-FR $\boldsymbol{\alpha}$-MNs. (B) Paired comparison shows the number of CTCs detected using anti-EpCAM-MNs alone or a combination of anti-EpCAM-MNs and anti-FR $\alpha-M N$. (C) Fluorescence microscopic images of cells isolated from patients with EOC and identified using three-color immunofluorescence staining. The cells were stained with Alexa Fluor ${ }^{\circledR} 568$-labeled anti-CK 19 monoclonal antibody (red), Alexa Fluor 488labeled anti-CD45 monoclonal antibody (green), and DAPI (blue). The Cytokeratin 19 (CK19)-positive (red), DAPI-positive (blue), and CD45-negative cells (green) indicated by the arrows were enumerated as CTCs. $* * * * \mathrm{P}<0.000 \mathrm{I}$. 
Table 2 Paired Chi-Square Test Of The Positive Rate When Using Anti-EpCAM-MNs Alone And That When Using A Combination Of Anti-EpCAM-MNs And Anti-FR-MNs In 95 Blood Samples From 30 Patients With Epithelial Ovarian Cancer

\begin{tabular}{|l|l|l|l|l|l|}
\hline \multicolumn{2}{|c|}{} & \multicolumn{2}{l|}{ Anti-EpCAM-MNs } & \multirow{2}{*}{$\chi^{2}$} \\
\cline { 3 - 5 } \multicolumn{2}{|l|}{} & CTC $\geq 2$ & CTC $<2$ & Total & \\
\hline Anti-EpCAM-MN and anti-FR $\alpha-M N$ & CTC $\geq 2$ & 45 & 19 & 64 & 14.45 \\
& CTC $<2$ & 1 & 30 & 31 & 0.000 \\
& Total & 46 & 49 & 95 & \\
\hline
\end{tabular}

Abbreviations: CTC, circulating tumor cells; EpCAM, epithelial cell adhesion molecule; FR $\alpha$, folate receptor alpha; MNs, magnetic nanospheres.

patients with benign disease is shown in Table 3 . The sensitivity and specificity of CTC detection using antiEpCAM-MNs were 0.536 and 0.9 , respectively, and that using the combination of anti-EpCAM-MNs and anti-FR $\alpha$ MNs was 0.75 and 0.85 , respectively. Thus, the combination of anti-EpCAM-MNs and anti-FR $\alpha$-MNs can improve the sensitivity of CTC detection in patients with newly diagnosed EOC $\left(\chi^{2}=4.17, \mathrm{P}=0.041\right)$. There was no statistical difference with respect to specificity. The Youden's index was 0.436 when using anti-EpCAM-MNs and 0.600 for the combination of anti-EpCAM-MNs and anti-FR $\alpha$-MNs.

\section{Discussion}

Previous studies have shown that CTCs from the same type of cancer and even from the same patient have extensive molecular and cellular heterogeneity. The CellSearch system remains the only CTC product approved by the FDA. However, because of high heterogeneity of cancer cells, clinical studies showed low sensitivity of EpCAM-based enrichment for CTC detection in patients with EOC. This was mainly due to EMT during metastasis, leading to the loss of more epithelium-like CTCs. FR $\alpha$ is highly expressed in ovarian cancer. It is a potential target for CTC capture of ovarian cancer, so we combined EpCAM and FR $\alpha$ as a capture target for CTCs to improve the detection rate of CTCs. First, in the mimic clinical sample, our results showed that the combined detection method can increase the capture rate of ovarian cancer CTC by capturing two target antigens. In the mixed cell samples of A2780 cells $\left(\mathrm{EpCAM}^{\text {low/ }}\right.$ $\left.\mathrm{FR} \alpha^{\text {high }}\right)$ and MCF7 cells $\left(\mathrm{EpCAM}^{\text {high }} / \mathrm{FR}^{\text {low }}\right.$ ) cells, the capture rate was more than $92.5 \%$ using a combination of anti-EpCAM-MNs and anti-FR $\alpha-M N s$, which exceeded the rate when using anti-EpCAM-MNs or anti-FR $\alpha$-MNs alone by approximately $20 \%(P<0.01)$. This result showed that the selection of different targets is important for capturing CTCs with different molecular expression characteristics. Studies have shown that there is an increase in the expression of FR $\alpha$ in tumor cells with low expression of EpCAM, ${ }^{12}$ which is the theoretical basis for the capture of more heterogeneous CTCs by EpCAM combined with FR $\alpha$ as a CTC capture target. EpCAM and FR $\alpha$ appeared to be complementary CTC capture targets, but due to the heterogeneity of the tumor, the combination of EpCAM and FR $\alpha$ may not be able to include all CTCs. More research will need to optimize the capture targets of CTC.

Table 3 Sensitivity And Specificity In 28 Patients With Initially Diagnosed Epithelial Ovarian Cancer And 20 Patients With Benign Disease

\begin{tabular}{|c|c|c|c|c|c|c|c|}
\hline \multicolumn{2}{|l|}{ Groups } & \multicolumn{3}{|l|}{ Patients } & \multicolumn{3}{|c|}{ Healthy Donor } \\
\hline & & \multicolumn{3}{|c|}{ Anti-EpCAM-MNs And Anti-FR $\alpha-M N s$} & \multicolumn{3}{|c|}{ Anti $\geq 2$-EpCAM-MNs And Anti-FR $\alpha-M N s$} \\
\hline & & CTC $\geq 2$ & CTC $<2$ & Total & СTC & CTC $<2$ & Total \\
\hline \multirow[t]{3}{*}{ Anti-EpCAM-MNs } & $\mathrm{CTC} \geq 2$ & 15 & 0 & 15 & 0 & 2 & 2 \\
\hline & CTC $<2$ & 6 & 7 & 13 & 3 & 15 & 18 \\
\hline & Total & 21 & 7 & 28 & 3 & 17 & 20 \\
\hline \multicolumn{2}{|l|}{ Groups } & \multicolumn{2}{|l|}{ Sensitivity } & \multicolumn{2}{|c|}{ Specificity } & \multicolumn{2}{|c|}{ Youden's index } \\
\hline \multirow{2}{*}{\multicolumn{2}{|c|}{$\begin{array}{l}\text { Anti-EpCAM-MNs and anti-FR } \alpha-M N s \\
\text { Anti-EDCAM-MNs }\end{array}$}} & \multicolumn{2}{|l|}{0.750} & \multicolumn{2}{|l|}{0.850} & \multicolumn{2}{|l|}{0.600} \\
\hline & & \multicolumn{2}{|l|}{0.536} & \multicolumn{2}{|l|}{0.900} & \multicolumn{2}{|l|}{0.436} \\
\hline
\end{tabular}

Abbreviations: CTC, circulating tumor cells; EpCAM, epithelial cell adhesion molecule; FR $\alpha$, folate receptor alpha; MNs, magnetic nanospheres. 
In the CTC detection of patients with 95 blood samples collected from 30 patients with EOC and 20 blood samples collected from 20 patients with ovarian benign disease, the results demonstrated that combined FR $\alpha$ as a capture target for immune capture based on EpCAM could significantly increase the capture rate of CTCs. When the threshold for positive CTCs in this study was $\geq 2$, the positive rate for the combined detection method was $67.36 \%$, whereas that for anti-EpCAM-MNs alone was $48.42 \%$, and this difference was statistically significant $\left(\chi^{2}=14.45, P<0.001\right)$. In patients with suspected EOC, the sensitivity of the combined detection method for CTCs was $75.0 \%$, which was significantly higher than that when using anti-EpCAM-MNs alone $\left(\chi^{2}=4.17, P=0.041\right)$. The area under the receiver operating characteristic curve (AUC-ROC) analysis indicated that the combination of anti-EpCAM-MNs and anti-FR $\alpha-\mathrm{MNs}$ had a higher AUC (0.8011, 95\% CI: 0.7212-0.8809, $P<0.001)$ than anti-EpCAM-MNs alone $(0.7208,95 \%$ CI: 0.6209 $0.8206, P=0.002)$, although the $t$-test showed no statistically significant difference between the two AUC $(P=0.12)$, the reason may be that the sample size is insufficient. The low detection rate of CTC detection using EpCAM as a capture target in patients with ovarian cancer in this study is consistent with multiple previous studies. A study used Cellsearch to capture CTC in 29 newly diagnosed ovarian cancer patients, only found that CTC can be detected in $17 \%$ of patients. ${ }^{13}$ Behbakht's research showed that CTC was enriched in 19/43 (44\%) blood specimens prior to the first cycle of chemotherapy in patients with ovarian cancer. ${ }^{14}$ Poveda's study showed that $14.4 \%(31 / 216)$ patients with ovarian cancer had 2 or more CTCs prior to the start of therapy. ${ }^{15}$ Liu's study found that when the CTC threshold was set to $\geq 2,60 \%(18 / 30)$ of ovarian cancer patients in the initial stage had CTC positive, and $58 \%(28 / 48)$ of the ovarian patients who relapsed had CTC positive. ${ }^{16}$

Intact CTCs can be used for determining functional cellular characteristics ${ }^{17}$ and performing molecular profiling at the DNA, RNA, and protein levels. ${ }^{18}$ This might guide personalized treatment selection, particularly after treatment failure. Moreover, CTC analysis enables multiple mutation detection within the same cell, thereby making mapping of the clonal evolution and tumor heterogeneity possible and unraveling potential associations between the mutational status and pathway activation by combining genomic and transcriptomic profiles of CTCs. ${ }^{19,20}$ Therefore, the CTC detection method should also include the step of nondestructive release. Our research revealed that after separation, CTCs were intact and viable. However, the nondestructive release of CTCs in our method requires further research because antibody/antigen interaction is strong and harsh treatment is needed to release the bound target. An emerging method using aptamers, which can be an alternative to CTC capture using antibodies, is currently under investigation. Some researchers have reported that aptamers can release CTCs without harsh procedures, ${ }^{21}$ such as the introduction of a complementary sequence to compete with binding with the target, ${ }^{22}$ removal via thermal denaturation, ${ }^{23}$ or wash steps using endonucleases. ${ }^{24}$ However, for antibody/antigen interactions, harsh treatment, such as enzyme degradation, ${ }^{25}$ is needed to release the bound target.

Although this study was able to capture $\mathrm{EpCAM}^{+}$cells and $\mathrm{FR}^{+}$cells, there may be some CTCs with phenotypic characteristic of $\mathrm{EpCAM}^{-} \mathrm{FR}^{-}$. Another potential limitation is that one $\mathrm{CK}_{1} 9^{+} / \mathrm{DAPI}^{+} / \mathrm{CD} 45^{-}$cell is detected in $8 \%$ of (8/95) ovarian cancer patients and $25 \%$ of healthy people, but it was not confirmed whether this one $\mathrm{CK}{ }^{+} / \mathrm{DAPI}^{+} /$ CD45 ${ }^{-}$cell was truly malignant or represented benign circulating epithelial cells. The possible reason may be that we did not strictly request to exclude the first tube blood sample during the collection process and may lead to the incorporation of vascular endothelial cells.

In summary, the results of this exploratory study show for the first time that the combined immunomagnetic separation technique of combined EpCAM and FR can be used to identify and count CTC in certain ovarian cancer patients. The combination of EpCAM and FR $\alpha$ as capture targets can significantly increase the CTC capture rate and prevent low EpCAM-expressing or EMT-converted CTCs from being missed. More experiments are required for subsequent analysis of $\mathrm{FR}^{+}$CTCs. Meanwhile, the number of tested samples and follow-up time are being studied to assess the correlation between $\mathrm{FR}^{+} \mathrm{CTCs}$ and clinical outcome.

\section{Conclusion}

The combined use of surface markers of EpCAM and FR $\alpha$ as CTC capture targets in EOC can improve the sensitivity of CTC detection. Captured CTCs were intact and viable, and they could be used in subsequent analyses, such as single-cell sequencing. Despite this promising result, further studies are required to assess the clinical validity of this method in patients with EOC.

\section{Acknowledgments}

The authors thank the reviewers for their helpful comments on this article. This study was funded by National 
Natural Science Foundation of China (NNSFC) (81802980, 81770169, and 81670144) and the Young Talent Foundation of Health and Family Planning Commission of Hubei Province (WJ2017Q007).

\section{Disclosure}

The authors report no conflicts of interest in this work.

\section{References}

1. Torre LA, Trabert B, DeSantis CE, et al. Ovarian cancer statistics, 2018. CA Cancer J Clin. 2018;68(4):284-296.

2. Giannopoulou L, Kasimir-Bauer S, Lianidou ES. Liquid biopsy in ovarian cancer: recent advances on circulating tumor cells and circulating tumor DNA. Clin Chem Lab Med. 2018;56(2):186-197. doi:10.1515/cclm-2017-0019

3. Dementeva N, Kokova D, Mayboroda OA. Current methods of the Circulating Tumor Cells (CTC) analysis: a brief overview. Curr Pharm Des. 2017;23(32):4726-4728. doi:10.2174/1381612823666170616082 608

4. Burinaru TA, Avram M, Avram A, et al. Detection of circulating tumor cells using microfluidics. ACS Comb Sci. 2018;20(3):107-126. doi:10.1021/acscombsci.7b00146

5. Rawal S, Ao Z, Datar RH, Agarwal A. Microfilter-based capture and release of viable circulating tumor cells. Methods Mol Biol. 2017;1634:93-105. doi:10.1007/978-1-4939-7144-2 7

6. Chen X, Zhou F, Li X, et al. Folate receptor-positive circulating tumor cell detected by LT-PCR-based method as a diagnostic biomarker for non-small-cell lung cancer. J Thorac Oncol. 2015;10 (8):1163-1171. doi:10.1097/JTO.0000000000000606

7. Kalli KR, Oberg AL, Keeney GL, et al. Folate receptor alpha as a tumor target in epithelial ovarian cancer. Gynecol Oncol. 2008;108 (3):619-626. doi:10.1016/j.ygyno.2007.11.020

8. Oseledchyk A, Andreou C, Wall MA, Kircher MF. Folate-targeted surface-enhanced resonance raman scattering nanoprobe ratiometry for detection of microscopic ovarian cancer. ACS Nano. 2017;11 (2):1488-1497. doi:10.1021/acsnano.6b06796

9. Chen L, Peng M, Li N, et al. Combined use of EpCAM and FRalpha enables the high-efficiency capture of circulating tumor cells in nonsmall cell lung cancer. Sci Rep. 2018;8(1):1188. doi:10.1038/s41598018-19391-1

10. Liu S, Tian Z, Zhang L, et al. Combined cell surface carbonic anhydrase 9 and CD147 antigens enable high-efficiency capture of circulating tumor cells in clear cell renal cell carcinoma patients. Oncotarget. 2016;7(37):59877-59891. doi:10.18632/oncotarget.10979

11. Weissenstein U, Schumann A, Reif M, Link S, Toffol-Schmidt UD, Heusser P. Detection of circulating tumor cells in blood of metastatic breast cancer patients using a combination of cytokeratin and EpCAM antibodies. BMC Cancer. 2012;12:206. doi:10.1186/14712407-12-206
12. Lou J, Ben S, Yang G, et al. Quantification of rare circulating tumor cells in non-small cell lung cancer by ligand-targeted PCR. PLoS One. 2013;8(12):e80458. doi:10.1371/journal.pone.0080458

13. Lou E, Vogel RI, Teoh D, et al. Assessment of circulating tumor cells as a predictive biomarker of histology in women with suspected ovarian cancer. Lab Med. 2018;49(2):134-139. doi:10.1093/labmed/ $\operatorname{lm} x 084$

14. Behbakht K, Sill MW, Darcy KM, et al. Phase II trial of the mTOR inhibitor, temsirolimus and evaluation of circulating tumor cells and tumor biomarkers in persistent and recurrent epithelial ovarian and primary peritoneal malignancies: a gynecologic oncology group study. Gynecol Oncol. 2011;123(1):19-26. doi:10.1016/j.ygyno.2011.06.022

15. Poveda A, Kaye SB, McCormack R, et al. Circulating tumor cells predict progression free survival and overall survival in patients with relapsed/recurrent advanced ovarian cancer. Gynecol Oncol. 2011;122(3):567-572. doi:10.1016/j.ygyno.2011.05.028

16. Liu JF, Kindelberger D, Doyle C, Lowe A, Barry WT, Matulonis UA. Predictive value of circulating tumor cells (CTCs) in newly-diagnosed and recurrent ovarian cancer patients. Gynecol Oncol. 2013;131(2):352-356. doi:10.1016/j.ygyno.2013.08.006

17. Baccelli I, Schneeweiss A, Riethdorf S, et al. Identification of a population of blood circulating tumor cells from breast cancer patients that initiates metastasis in a xenograft assay. Nat Biotechnol. 2013;31 (6):539-544. doi:10.1038/nbt.2576

18. Heitzer E, Auer M, Gasch C, et al. Complex tumor genomes inferred from single circulating tumor cells by array-CGH and next-generation sequencing. Cancer Res. 2013;73(10):2965-2975. doi:10.1158/ 0008-5472.CAN-12-4140

19. Brouwer A, De Laere B, Peeters D, et al. Evaluation and consequences of heterogeneity in the circulating tumor cell compartment. Oncotarget. 2016;7(30):48625-48643. doi:10.18632/oncotarget.8015

20. Van Berckelaer C, Brouwers AJ, Peeters DJ, Tjalma W, Trinh XB, van Dam PA. Current and future role of circulating tumor cells in patients with epithelial ovarian cancer. Eur J Surg Oncol. 2016;42 (12):1772-1779. doi:10.1016/j.ejso.2016.05.010

21. Zamay AS, Zamay GS, Kolovskaya OS, Zamay TN, Berezovski MV. Aptamer-based methods for detection of circulating tumor cells and their potential for personalized diagnostics. Adv Exp Med Biol. 2017;994:67-81. doi:10.1007/978-3-319-55947-6 3

22. Zhang Z, Chen N, Li S, Battig MR, Wang Y. Programmable hydrogels for controlled cell catch and release using hybridized aptamers and complementary sequences. J Am Chem Soc. 2012;134(38):1571615719. doi:10.1021/ja307717w

23. Zhu J, Nguyen T, Pei R, Stojanovic M, Lin Q. Specific capture and temperature-mediated release of cells in an aptamer-based microfluidic device. Lab Chip. 2012;12(18):3504-3513. doi:10.1039/c2lc $40411 \mathrm{~g}$

24. Nair SV, Witek MA, Jackson JM, et al. Enzymatic cleavage of uracilcontaining single-stranded DNA linkers for the efficient release of affinity-selected circulating tumor cells. Chem Commun. 2015;51 (15):3266-3269. doi:10.1039/c4cc09765c

25. Sheng W, Ogunwobi OO, Chen T, et al. Capture, release and culture of circulating tumor cells from pancreatic cancer patients using an enhanced mixing chip. Lab Chip. 2014;14(1):89-98. doi:10.1039/ c31c51017d
Cancer Management and Research is an international, peer-reviewed open access journal focusing on cancer research and the optimal use of preventative and integrated treatment interventions to achieve improved outcomes, enhanced survival and quality of life for the cancer patient.
The manuscript management system is completely online and includes a very quick and fair peer-review system, which is all easy to use. Visit http://www.dovepress.com/testimonials.php to read real quotes from published authors. 